\title{
Dynamic insulin sensitivity index: importance in
}

diabetes

Gianluigi Pillonetto, Andrea Caumo and Claudio Cobelli

Am J Physiol Endocrinol Metab 298:E440-E448, 2010. First published 17 November 2009;

doi:10.1152/ajpendo.90340.2008

You might find this additional info useful...

This article cites 16 articles, 9 of which can be accessed free at:

http://ajpendo.physiology.org/content/298/3/E440.full.html\#ref-list-1

Updated information and services including high resolution figures, can be found at:

http://ajpendo.physiology.org/content/298/3/E440.full.html

Additional material and information about AJP - Endocrinology and Metabolism can be found at: http://www.the-aps.org/publications/ajpendo

This infomation is current as of August 16, 2011.

AJP - Endocrinology and Metabolism publishes results of original studies about endocrine and metabolic systems on any level of organization. It is published 12 times a year (monthly) by the American Physiological Society, 9650 Rockville Pike, Bethesda MD 20814-3991. Copyright @ 2010 by the American Physiological Society. ISSN: 0193-1849, ESSN: 1522-1555. Visit our website at http://www.the-aps.org/. 


\title{
Dynamic insulin sensitivity index: importance in diabetes
}

\author{
Gianluigi Pillonetto, ${ }^{1}$ Andrea Caumo, ${ }^{2}$ and Claudio Cobelli ${ }^{1}$ \\ ${ }^{1}$ Dipartimento di Ingegneria dell'Informazione, Università degli Studi di Padova, Padova; and ${ }^{2}$ San Raffaele Scientific \\ Institute, Milan, Italy
}

Submitted 7 April 2008; accepted in final form 12 November 2009

Pillonetto G, Caumo A, Cobelli C. Dynamic insulin sensitivity index: importance in diabetes. Am J Physiol Endocrinol Metab 298: E440-E448, 2010. First published November 17, 2009; doi:10.1152/ajpendo.90340.2008.-The classical minimal model (MM) index of insulin sensitivity, $\mathrm{S}_{\mathrm{I}}$, does not account for how fast or slow insulin action takes place. In a recent work, we proposed a new dynamic insulin sensitivity index, $S_{I}^{\mathrm{D}}$, which is able to take into account the dynamics of insulin action as well. The new index is a function of two MM parameters, namely $S_{I}$ and $p_{2}$, the latter parameter governing the speed of rise and decay of insulin action. We have previously shown that in normal glucose tolerant subjects $S_{I}^{D}$ provides a more comprehensive picture of insulin action on glucose metabolism than $S_{I}$. The aim of this study is to show that resorting to $S_{I}^{D}$ rather $S_{I}$ is even more appropriate when studying diabetic patients who have a low and slow insulin action. We analyzed insulinmodified intravenous glucose tolerance test studies performed in 10 diabetic subjects and mixed meal glucose tolerance test studies exploiting the triple tracer technique in 14 diabetic subjects. We derived both $S_{I}$ and $S_{I}^{D}$ resorting to Bayesian and Fisherian identification strategies. The results show that $S_{I}^{D}$ is estimated more precisely than $\mathrm{S}_{\mathrm{I}}$ when using the Bayesian approach. In addition, the less laborintensive Fisherian approach can still be used to obtain reliable point estimates of $S_{I}^{D}$ but not of $S_{I}$. These results suggest that $S_{I}^{D}$ yields a comprehensive, precise, and cost-effective assessment of insulin sensitivity in subjects with impaired insulin action like impaired glucose tolerant subjects or diabetic patients.

model; insulin resistance; parameter estimation; Bayesian estimation; Markov chain Monte Carlo strategy; diabetes

THE MINIMAL MODEL (MM) of glucose kinetics has been used in nearly 600 papers since its inception in the late seventies (4). The reason for such popularity is related to the ability of MM to provide an index of insulin sensitivity, denoted by $\mathrm{S}_{\mathrm{I}}$, from the analysis of an intravenous glucose tolerance test (IVGTT). An extension of this model is being currently used also to determine $S_{\text {I }}$ from an oral/meal glucose tolerance test (OGTT/ MGTT; Ref. 8).

Albeit of paramount importance, it must be recognized that $\mathrm{S}_{\mathrm{I}}$ does not yield an exhaustive picture of insulin action. The reason can be more easily grasped by making reference to the assessment of insulin sensitivity with the gold standard method, which is the euglycemic hyperinsulinemic clamp. The clamp-based insulin sensitivity, of which the MM-based $\mathrm{S}_{\mathrm{I}}$ is a theoretical counterpart, is measured at the end of the clamp when insulin action is at steady state. However, in those clamp studies that monitored in detail the time course of insulin action from the initial to the final steady state, it became apparent that the speed of rise of insulin action varied considerably among individuals and that such speed was related to the degree of

Address for reprint requests and other correspondence: C. Cobelli, Dipartimento di Ingegneria dell'Informazione, Università degli Studi di Padova, Via Gradenigo, 6/B-35131 Padova, Italy (e-mail: cobelli@dei.unipd.it). insulin sensitivity measured at steady state (15). This observation indicates that when judging on the individual's ability to dispose glucose, not only is the maximum excursion of insulin action relevant, but also the dynamics of insulin action plays a role. If this is true during a clamp, a fortiori is true during an IVGTT in which insulin action never attains a plateau and its speed of rise and decay certainly influence the return of glucose to the baseline. For this reason, we proposed a new insulin sensitivity index that, at variance with the classic one, also accounts for the dynamics of insulin action. The new index, denoted by $\mathrm{S}_{\mathrm{I}}^{\mathrm{D}}$, is able to account for both the speed and the capacity of response. Thanks to this peculiarity, in Ref. 17 we have shown that in normal glucose tolerant (NGT) subjects $S_{I}^{D}$ provides, compared with $\mathrm{S}_{\mathrm{I}}$, a more comprehensive picture of insulin action on glucose metabolism. In addition, $S_{I}^{D}$ is intrinsically more robust than $S_{I}$, which makes its identification easier and more reliable with respect to $S_{I}$.

The aim of the present study is to compare the estimation of $S_{I}$ and $S_{I}^{D}$ in diabetic patients and verify whether in these subjects the assessment of $\mathrm{S}_{\mathrm{I}}^{\mathrm{D}}$ produces even greater benefits than in NGT subjects. There are two reasons for this to happen. The first one is related to the notion that not only are diabetic patients insulin resistant, but, according to the above-mentioned euglycemic hyperinsulinemic studies, their insulin action develops at a slower pace than in NGT subjects $(1,15)$. As a result, in diabetic patients $S_{I}^{D}$ should be more capable than $S_{I}$ to provide a realistic picture of how a diabetic patient is able to handle the glucose challenge. Moreover, $S_{I}^{D}$ should be more accurate than $S_{I}$ in ranking diabetic patients. The second reason why $S_{I}^{D}$ is expected to perform better than $S_{I}$ in diabetic patients is related to the numerical identifiability of the MM in such patients. It is well known that in diabetics the MM identification is difficult because it is often associated with large parameters uncertainties (see Refs. 1, 18, 19, 21). The greater robustness of $\mathrm{S}_{\mathrm{I}}^{\mathrm{D}}$ with respect to $\mathrm{S}_{\mathrm{I}}$ should ensure that $\mathrm{S}_{\mathrm{I}}^{\mathrm{D}}$ is precisely estimated even in those patients in which the estimation of $\mathrm{S}_{\mathrm{I}}$ is difficult or impossible.

\section{MATERIALS AND METHODS}

Estimates of $S_{I}$ and $S_{I}^{D}$ were obtained in diabetic patients under two different experimental conditions: an insulin-modified IVGTT and a MGTT. The IVGTT studies were performed in 10 type 2 diabetic patients whose characteristics have been already described in Refs. 1 and 19. Briefly, the IVGTT consisted of a glucose dose of $300 \mathrm{mg} / \mathrm{kg}$ injected at time 0 followed by a short insulin infusion $(0.05 \mathrm{U} / \mathrm{kg})$ administered between 20 and $25 \mathrm{~min}$. The MGTT studies were performed in 14 type 2 diabetic subjects who underwent a mixed meal test in which three tracers were simultaneously infused to allow an accurate determination of the rate of appearance of the orally administered glucose into the circulation (see Ref. 3 for details about the experimental protocol and the subjects' characteristics). The protocol was approved by The Mayo Clinic Institutional Review Board. 
To compare the ability of $S_{I}$ and $S_{I}^{D}$ to discriminate between diabetic and NGT subjects, we performed a simulation study. Estimates of $S_{I}$ and $S_{I}^{D}$ were obtained from synthetic insulin modified IVGTT experiments performed in 1,000 diabetic and 1,000 NGT subjects. Synthetic data were generated using two log-normal priors for MM parameters. The first prior was obtained from the estimates in diabetic subjects reported in Table 1, while the second prior was derived from the estimates obtained in 10 NGT individuals in a previous study (details are reported in Ref. 17). Then, the typical insulin profile that can be observed during an insulin-modified IVGTT in a diabetic subject was used as the insulin input to the model. We sampled all the generated glucose profiles at 5, 8, 10, 12, 15, 20, 25, $30,40,60,80,100,120,140,160,180$, and $240 \mathrm{~min}$ and finally corrupted them by white normal noise with a coefficient of percentage variation equal to 2 .

To validate the new insulin sensitivity index, we used correlation analysis to compare the estimates of $\mathrm{S}_{\mathrm{I}}^{\mathrm{D}}$ obtained in 21 subjects [11 impaired glucose tolerant (IGT) and 10 NGT] undergoing both an OGTT and a euglycemic hyperinsulinemic clamp. Details about the experimental procedures are reported in the Methods in Ref. 9. Here, we just recall that, during the clamp, regular human insulin was infused at a constant rate $\left(25 \mathrm{mU} \cdot \mathrm{m}^{-2} \cdot \mathrm{min}^{-1}\right)$ for $180 \mathrm{~min}$. A hyperinsulinemic plateau level was achieved within 30-45 min from the initiation of the insulin infusion. Due to the natural variability of the insulin clearance among subjects, the hyperinsulinemic plateau level differed slightly among individuals (means \pm SD close to 50 and $10 \mu \mathrm{U} / \mathrm{ml}$, respectively). However, the clamp-based index of insulin sensitivity $\left[\mathrm{S}_{\mathrm{I}}\right.$ (clamp) and the new $\mathrm{S}_{\mathrm{I}}^{\mathrm{D}}$ (clamp)], as well as the MMbased indices $S_{I}$ and $S_{I}^{D}$, have the intrinsic ability to properly account for different hyperinsulinemic levels among individuals (e.g., see Eqs. 2 and 5).

MM and derivation of dynamic insulin sensitivity. In the following we refer, without loss of generality, to the MM equations describing glucose disappearance during an IVGTT. The equations describing glucose disappearance during an MGTT are the same with the only difference that the exogenous glucose input is the rate of appearance of orally administered glucose that has been accurately estimated thanks to the triple-tracer technique (see Ref. 3 for details). MM equations are as follows:

$$
\begin{array}{cl}
\dot{\mathrm{G}}(t)=-\left(p_{1}+X(t)\right) \mathrm{G}(t)+\mathrm{p}_{1} \mathrm{G}_{\mathrm{b}} & \mathrm{G}(0)=\mathrm{G}_{0} \\
\dot{X}(t)=-p_{2} X(t)+p_{3}\left(\mathrm{I}(t)-\mathrm{I}_{\mathrm{b}}\right) & X(0)=0
\end{array}
$$

where $\mathrm{G}(t)(\mathrm{mg} / \mathrm{dl})$ and $\mathrm{I}(t)(\mu \mathrm{U} / \mathrm{ml})$ are glucose and insulin concentrations in plasma, respectively, while $G_{b}$ and $I_{b}$ are their baseline values. Equation 1 represents glucose kinetics, whereas Eq. 2 describes insulin action exerted from a compartment remote from plasma. Uniquely identifiable model parameters are $p_{1}, p_{2}, p_{3}$, and $\mathrm{G}_{0}$. Parameters of interest provided by the model are glucose effectiveness $\left(\mathrm{S}_{\mathrm{G}}=p_{1}, \min ^{-1}\right)$, which measures the effect of glucose per se on both glucose disappearance and endogenous glucose production, and insulin sensitivity $\left(\mathrm{S}_{\mathrm{I}}=p_{3 /} p_{2}, \min ^{-1} \mu \mathrm{U}^{-1} \mathrm{ml}\right)$, which measures insulin's ability to enhance glucose effectiveness by both increasing glucose disappearance and inhibiting endogenous glucose production. Parameter $p_{2}$ is the time constant of the remote insulin compartment and thus governs the speed of rise and decay of insulin action. Parameters $\mathrm{S}_{\mathrm{I}}$ and $p_{2}$ can be used to derive a dynamic index of insulin sensitivity, as already demonstrated in Ref. 17. For the sake of clarity and completeness, we will briefly state without proof the steps leading to the derivation of $S_{\mathrm{I}}^{\mathrm{D}}$. The first step consists of obtaining a closed form of the MM glucose prediction, as a function of model parameters and insulin profile. This aim can be accomplished by noticing that $E q .2$ can be integrated independently from $E q$. 1 . We use $Z(t)$ to denote the integral function of remote insulin. We see from Eq. 2 that the impulse response relating the deviation of insulin from basal and $X(t)$ is $p_{3} e^{-p_{2} \sigma}=$ $\mathrm{S}_{\mathrm{I}} p_{2} e^{-p_{2} \sigma}$ for $\sigma \geq 0$ and zero otherwise. Thus $Z(t)$ is the output of a system with impulse response

$$
\begin{aligned}
& \int_{0}^{t} \mathrm{~S}_{\mathrm{I}} p_{2} e^{-\sigma p_{2}} d \sigma=-\mathrm{S}_{\mathrm{I}} e^{-\left.\sigma p_{2}\right|_{0} ^{t}}=\mathrm{S}_{\mathrm{I}}\left(1-e^{-p_{2} t}\right), \mathrm{i} . e . \\
& Z(t)=\int_{0}^{t} X(\sigma) d \sigma=\mathrm{S}_{\mathrm{I}} \int_{0}^{t}\left(1-e^{-p_{2}(t-\sigma)}\right)\left(\mathrm{I}(\sigma)-\mathrm{I}_{\mathrm{b}}\right) d \sigma
\end{aligned}
$$

From a standard result on linear differential equations (see Ref. 6) glucose prediction is given by

$$
\mathrm{G}(t)=\mathrm{G}_{0} e^{-\mathrm{S}_{\mathrm{G}} t-Z(t)}+\mathrm{S}_{\mathrm{G}} \mathrm{G}_{\mathrm{b}} \int_{0}^{t} e^{-\mathrm{S}_{\mathrm{G}}(t-\tau)-Z(t)+Z(\tau)} d \tau
$$

Equation 4 allows one to appreciate that, according to the MM, glucose concentration is a function of two signals, i.e., $\mathrm{S}_{\mathrm{G}} t$ and $Z(t)$. The term $\mathrm{G}_{0} e^{-S_{\mathrm{G}} t-Z(t)}$ is a single decaying exponential with a coefficient proportional to the sum of these two signals. In particular, the first of these two signals, i.e., $\mathrm{S}_{\mathrm{G}} t$, is linearly dependent on glucose effectiveness, $\mathrm{S}_{\mathrm{G}}$. The second signal, i.e., $Z(t)$, describes the ability of insulin to enhance glucose effectiveness and depends in a more complex way on both $\mathrm{S}_{\mathrm{I}}$ and $p_{2}$. In fact, from $E q .3$ one can see that $Z(t)$ is the output of a time-invariant linear system having $\mathrm{I}(t)-\mathrm{I}_{\mathrm{b}}$ as input and $\mathrm{h}(t)=\mathrm{S}_{\mathrm{I}}\left(1-e^{-p_{2} t}\right)$ as unit impulse response. This impulse response, denoted by integrated insulin action impulse response (IAIR; Refs. 17, 18), describes the modalities by which incremental (above basal) insulin concentration is able to influence the glucose profile. Dynamic insulin sensitivity, $\mathrm{S}_{\mathrm{I}}^{\mathrm{D}}$, is defined as the mean value of the IAIR in a time interval of duration $T$ (how $T$ is chosen will be clarified in a moment). In mathematical terms, we have:

$$
\mathrm{S}_{\mathrm{I}}^{\mathrm{D}}=\frac{\int_{0}^{T} \mathrm{~S}_{\mathrm{I}}\left(1-e^{-p_{2} t}\right) d t}{T}=\mathrm{S}_{\mathrm{I}}\left[1-\frac{1-e^{-p_{2} T}}{p_{2} T}\right]
$$

\begin{tabular}{|c|c|c|c|c|c|}
\hline Subject Nos. & $\mathrm{S}_{\mathrm{I}}, 10^{-4} \min ^{-1} \mu \mathrm{U}^{-1} \mathrm{ml}$ & $p_{2}, 10^{-2} \min ^{-1}$ & $\mathrm{~S}_{\mathrm{I}}^{\mathrm{D}}, 10^{-4} \min ^{-1} \mu \mathrm{U}^{-1} \mathrm{ml}$ & $\mathrm{S}_{\mathrm{G}}, 10^{-2} \min ^{-1}$ & $\mathrm{G}_{0}, \mathrm{mg} / \mathrm{dl}$ \\
\hline 2 & $1.5(1.4-1.6)$ & $7(5.7-84)$ & $1.17(1.1-1.23)$ & $2.1(1.8-2.38)$ & $382(369.9-394.7)$ \\
\hline 3 & $1.7(0.4-4.1)$ & $0.18(0.04-0.68)$ & $0.06(0.028-0.1)$ & $1.06(0.78-1.35)$ & 434.6 (421.9-448.4) \\
\hline 5 & $2.1(1.8-2.2)$ & $9.8(7.8-12)$ & $1.7(1.52-1.91)$ & $0.89(0.48-1.32)$ & $411(395.6-428.4)$ \\
\hline 6 & $0.39(0.032-2.3)$ & $0.7(0.014-7)$ & $0.02(0.006-0.058)$ & $1.35(1-1.62)$ & $563(543.3-581.3)$ \\
\hline 7 & $1.51(0.36-4.1)$ & $0.19(0.033-0.8)$ & $0.05(0.03-0.087)$ & $1.48(1.32-1.62)$ & $314(304.8-323.5)$ \\
\hline 10 & $0.6(0.46-0.72)$ & $2.5(2.1-3)$ & $0.29(0.22-0.36)$ & $0.84(0.52-1.18)$ & $263(252.5-275.2)$ \\
\hline
\end{tabular}

It can be verified that $S_{I}^{D}$ is always less or equal to $S_{I}$. Thus $S_{I}^{D}$ can be conveniently expressed as a fraction, comprised between 0 and 1 , of $\mathrm{S}_{\mathrm{I}}$. Such fraction is denoted as efficiency:

Table 1. IVGTT in diabetic subjects: Bayesian estimates of minimal model parameters

Nos. in parentheses show $95 \%$ confidence intervals. IVGTT, intravenous glucose tolerance test; $\mathrm{S}_{\mathrm{I}}$, insulin sensitivity; $p_{2}$, rate parameter, $\mathrm{S}_{\mathrm{I}}^{\mathrm{D}}$, dynamic insulin sensitivity index; $\mathrm{S}_{\mathrm{G}}$, glucose effectiveness, $\mathrm{G}_{0}$, glucose at time 0 . 


$$
\eta\left(p_{2}, T\right)=\frac{\mathrm{S}_{\mathrm{I}}^{\mathrm{D}}}{\mathrm{S}_{\mathrm{I}}}=\left[1-\frac{1-e^{-p_{2} T}}{p_{2} T}\right]
$$

Efficiency $\eta$ measures which fraction of the potentially available insulin sensitivity, denoted by $S_{I}$, is translated into effective glycemic control by means of a rapid increase of insulin action. The rapidity of insulin action is governed by parameter $p_{2}$ and $\eta$ increases monotonically as a function of $p_{2}$ (once $T$ has been fixed). Notice that the closer $\eta$ to 1 , the larger the efficiency of the metabolic system in converting $S_{\text {I }}$ into an effective insulin control on glucose. Conversely, a very low $\eta$ renders $S_{I}^{D}$ close to zero, i.e., in this case insulin action is virtually unavailable during the experiment. This observation explains why a high $S_{I}$ value does not necessarily imply an effective control of insulin on glucose, since it can be associated with a low $p_{2}$ and thus with a low efficiency.

As far as the choice of $T$ is concerned, such a parameter does not represent the length of the IVGTT but is the duration of a "thought experiment" where insulin action has time to develop and IAIR can be directly observed. The "optimal" value of $T$ should allow $\eta$ to vary in the largest possible range of values so as to magnify differences between $\eta$ values of the subjects under study. The reader is referred to Ref. 17 for all the details. Here, we just recall that after considering a wide population of NGT, IGT, and diabetic individuals, a robust choice consists of setting $T$ to $60 \mathrm{~min}$.

MM identification: IVGTT data. The four unknown MM parameters that are estimated from IVGTT data are hereby defined by the parameter vector $\theta=\left[\mathrm{S}_{\mathrm{I}}, \mathrm{S}_{\mathrm{G}}, p_{2}, \mathrm{G}_{0}\right]$. These parameters are a priori uniquely identifiable given $\mathrm{G}(t)$ and $\mathrm{I}(t)$. As usual, we assume that $\mathrm{I}(t)$ in $E q .2$ is known at any $t$ by linearly interpolating its measured plasma concentration samples. $\mathrm{G}(t)$ is instead known in sampled and noisy form on a grid $\left(t_{1}, t_{2}, \ldots t_{\mathrm{N}}\right)$. The measurement error is a zero-mean Gaussian with uncorrelated components and a $2 \%$ coefficient of variation.

The MM parameters were estimated using either a Bayesian or a Fisherian approach. The Bayesian approach (10) was implemented by using the Markov chain Monte Carlo (MCMC) strategy (11, 13), already used in Refs. 17, 18, 19. This powerful approach allows one to reconstruct (in sampled form) the entire a posteriori probability density function of $\mathrm{S}_{\mathrm{I}}^{\mathrm{D}}$ and $\mathrm{S}_{\mathrm{I}}$, thus permitting a more effective comparison of the performance of the new index with respect to the classic one. The Bayesian approach requires a probabilistic description of the a priori knowledge concerning the parameters to be estimated. The $\mathrm{MM}$ parameters $\mathrm{S}_{\mathrm{I}}, \mathrm{S}_{\mathrm{G}}, p_{2}$, and $\mathrm{G}_{0}$ were assumed independent of each other and since all of them are nonnegative, priors whose support extends only in the positive axis were used. The a priori probability density functions of $\mathrm{S}_{\mathrm{G}}$ and $\mathrm{G}_{0}$ were assumed to be uniform in $[0, a]$ with $a \rightarrow+\infty$. The choice of the a priori probability density function of $S_{I}$ was based on the information on diabetic subjects reported in literature, e.g., Refs. 16 and 19. Specifically, we assumed for $S_{I}$ an a priori probability density function where $S_{I}$ values $<2 \times 10^{-4}\left(\mathrm{~min}^{-1} / \mu \mathrm{Uml}\right)$ are equally probable, whereas $\mathrm{S}_{\mathrm{I}}$ values $>2 \times 10^{-4}$ are less and less probable according to a decreasing exponential law (with exponent equal to $10^{4} \mu \mathrm{Uml}^{-1} / \mathrm{min}$ ). The choice of the a priori probability density function of $p_{2}$ was based on the rationale described in Ref. 18; that is, we assumed a uniform distribution in the interval $[0,5] \mathrm{min}^{-1}$. It is worth anticipating that the prior information on the MM parameters did not influence the general conclusions obtained in the study.

The MM was also identified using a Fisher parameter estimation scheme, which, albeit less sophisticated than the Bayesian one, makes it possible the adoption of mode-finding algorithms that are much less demanding than MCMC. This can have practical relevance in largescale or epidemiological studies in which $\mathrm{S}_{\mathrm{I}}^{\mathrm{D}}$ has to be estimated in many subjects. To carry out the Fisherian estimation of the MM parameters, a maximum likelihood (ML) estimator $(2,7)$ was adopted.

MM identification: MGTT data. As already observed in Ref. 3, when the MM is identified from MGTT data, some difficulties arise because the slow changes that glucose and insulin concentrations exhibit under postprandial conditions make it difficult to distinguish the individual contribution of insulin sensitivity and glucose effectiveness to glucose disappearance. To overcome this drawback, we followed the same strategy that was adopted in Ref. 3 to improve the numerical identifiability of the model, i.e., we exploited the fact that $\mathrm{S}_{\mathrm{G}}$, measuring glucose effectiveness at basal insulin, can be factored out in the sum of the glucose effectiveness at zero insulin (GEZI) and the product of insulin sensitivity times basal insulin concentration:

$$
\mathrm{S}_{\mathrm{G}}=\mathrm{GEZI}+\mathrm{S}_{\mathrm{I}} \mathrm{I}_{\mathrm{b}}
$$

The advantage of expressing $\mathrm{S}_{\mathrm{G}}$ in these terms lies in the possibility of introducing some reasonable assumptions about GEZI that guarantee an easier numerical identification of the MM. GEZI was assumed Gaussian with a mean of 0.025 and SD of 0.0025 . The MM parameters were estimated by maximizing a penalized likelihood accounting for Eq. 7 and the prior on GEZI. To investigate the sensitivity of the MM estimates to the prior on GEZI, the MM identification was also carried out using a much less informative prior on GEZI (the SD of GEZI was increased from 0.0025 to 0.01 ).

Estimation of $S_{I}^{D}$ from an OGTT and a euglycemic hyperinsulinemic clamp. We assessed the validity of $S^{D}$ by comparing its estimates obtained in 21 subjects who underwent both an OGTT and a euglycemic hyperinsulinemic clamp.

OGTT-based estimates of $\mathrm{S}_{\mathrm{I}}^{\mathrm{D}}$ were computed from Eq. 5 using the estimates of $\mathrm{S}_{\mathrm{I}}$ and $p_{2}$ obtained in Ref. 9. Of note is that the OGTT-based $\mathrm{S}_{\mathrm{I}}^{\mathrm{D}}$ estimate also accounts for the glucose distribution volume, V (see Eq. 7 in Ref. 9).

For what concerns computation of $S_{\mathrm{I}}^{\mathrm{D}}$ (clamp), we simply refer to the theoretical definition of $S_{1}^{D}$. During an ideal clamp, the glucose infusion rate, $R(t)$, is proportional to IAIR (see Appendix of Ref. 17) and equals insulin action multiplied by the distribution volume. Thus from $E q .2$ we have:

$$
\dot{R}(t)=-p_{2} R(t)+\mathrm{V} \times p_{3}\left(\mathrm{I}(t)-\mathrm{I}_{\mathrm{b}}\right) \quad \mathrm{R}(0)=0
$$

Parameters $p_{2}$ and $\mathrm{V} \times p_{3}$ governing the dynamics of $R(t)$ were estimated by fitting the samples of $R(t)$ via nonlinear least squares (the forcing input was defined by linearly interpolating the samples of insulin collected during the clamp). Finally, the dynamic insulin sensitivity was computed as follows:

$$
\mathrm{S}_{\mathrm{I}}^{\mathrm{D}}(\text { clamp })=\frac{\mathrm{V} p_{3}}{p_{2}}\left[1-\frac{1-e^{-p_{2} T}}{p_{2} T}\right] \text { with } T=60 \mathrm{~min}
$$

\section{RESULTS}

The MM identification results of the 10 diabetic patients who underwent the IVGTT are reported in Table 1 (Bayesian approach) and Table 2 (Fisherian approach). In particular, Table 1 reports the minimum variance estimates of $\mathrm{S}_{\mathrm{I}}, p_{2}, \mathrm{~S}_{\mathrm{I}}^{\mathrm{D}}$, $\mathrm{S}_{\mathrm{G}}$, and $G_{0}$, and the $95 \%$ confidence intervals (obtained as the

Table 2. IVGTT in diabetic subjects: maximum likelihood $S_{I}$ and $S_{I}^{D}$ estimates

\begin{tabular}{ccc}
\hline \hline Subject Nos. & $\mathrm{S}_{\mathrm{I}}, 10^{-4} \mathrm{~min}^{-1} \mu \mathrm{U}^{-1} \mathrm{ml}$ & $\mathrm{S}_{\mathrm{I}}^{\mathrm{D}}, 10^{-4} \mathrm{~min}^{-1} \mu \mathrm{U}^{-1} \mathrm{ml}$ \\
\hline 1 & 0.92 & 0.31 \\
2 & 1.51 & 1.16 \\
3 & 0.22 & 0.029 \\
4 & 0.86 & 0.15 \\
5 & 2.1 & 1.74 \\
6 & 0.14 & 0.027 \\
7 & 0.98 & 0.046 \\
8 & 0.99 & 0.935 \\
9 & 0.52 & 0.017 \\
10 & 0.58 & 0.29 \\
\hline
\end{tabular}


interval between quantiles 2.5 and $97.5 \%$ ) in parentheses. Table 2 shows the ML estimates of $S_{I}$ and $S_{I}^{D}$. Figure 1 shows the marginal posteriors (obtained in sampled form by MCMC) of the parameters $S_{\mathrm{I}}, p_{2}$, and $\mathrm{S}_{1}^{\mathrm{D}}$ for subjects 2 (top) and 3 (bottom). Figure 2 displays $S_{I}$ and $S_{I}^{D}$ point estimates obtained in all the 10 subjects with $95 \%$ confidence intervals. Figure 3 compares $\mathrm{S}_{\mathrm{I}}$ (left) and $\mathrm{S}_{\mathrm{I}}^{\mathrm{D}}($ right $)$ estimates obtained using the Fisherian and the Bayesian approach. The correlation coefficient between ML and Bayes estimates of $\mathrm{S}_{\mathrm{I}}$ and of $\mathrm{S}_{\mathrm{I}}^{\mathrm{D}}$ turns out to be 0.67 and 0.99 , respectively.

Table 3 reports the identification results of the 14 diabetic patients who underwent the MGTT. In this table, the estimates of $\mathrm{S}_{\mathrm{I}}, p_{2}$, and $\mathrm{S}_{\mathrm{I}}^{\mathrm{D}}$ obtained using both the full as well as the less informative prior on GEZI are shown. Figure 4 compares the $S_{I}$ (left) and $\mathrm{S}_{\mathrm{I}}^{\mathrm{D}}(r i g h t)$ estimates obtained with the two priors.

Figure 5 reports the results of the simulation study designed to compare the performance of $S_{I}$ and $S_{I}^{D}$ in NGT and diabetic subjects. The true values of $S_{I}$ and $S_{I}^{D}$ are plotted against the values estimated by ML in the two groups. The correlation coefficients between true and estimated $\mathrm{S}_{\mathrm{I}}$ are $\sim 0.1$ and 0.4 in diabetic and NGT subjects, respectively, while those concerning $S_{I}^{\mathrm{D}}$ are 0.98 and 0.99 , respectively.

Figures 6 and 7 concern the results obtained in the 21 subjects who underwent both an OGTT and a clamp experiment. Figure 6 shows the fit of clamp data in one representative IGT subject: the glucose infusion rate values during the clamp are denoted by the open circles, while the solid line describes the curve, proportional to IAIR, obtained after fitting the samples using Eq. 8 and nonlinear least squares. The area under the curve in the first $60 \mathrm{~min}$ (shaded area) is proportional to $S_{1}^{\mathrm{D}}$ (clamp). Figure 7 compares the $S_{1}^{\mathrm{D}}$ (clamp) and $S_{1}^{\mathrm{D}}$ estimates obtained in the two experimental situations via correla- tion analysis. In particular, the correlation coefficient between OGTT-based estimate of $S_{1}^{D}$ and $S_{1}^{D}$ (clamp) is 0.82 .

\section{DISCUSSION}

IVGTT in diabetic subjects: $S_{I}$ vs. $S_{I}^{D}$ using Bayes estimation. To comment on the results of the Bayesian estimation, it is helpful to examine the MM identification results obtained in two paradigmatic subjects. Figure 1 plots the marginal posteriors (obtained in sampled form by MCMC) of the parameters $\mathrm{S}_{\mathrm{I}}, p_{2}$, and $\mathrm{S}_{\mathrm{I}}^{\mathrm{D}}$ for subject 2 (top) and 3 (bottom). As far as $\mathrm{S}_{\mathrm{I}}$ is concerned (first column of Fig. 1), one can see that this parameter is well estimated in subject 2. In fact, its posterior appears well concentrated around its mean. From Table 1, one can see that the point estimate of $S_{I}$ is $1.5(1.4-1.6)$ in this subject. The situation is completely different for subject 3 . In this subject, $S_{I}$ suffers of poor numerical identifiability, as indicated by its long-tailed marginal posterior (this phenomenon is mathematically explained by Proposition 2 in Ref. 18). In addition, the point estimate of $S_{I}$ is $1.7(0.4-4.1)$ and thus very close to the mean of the prior (whose value is $\sim 1.67$ ). This suggests that the IVGTT data provide little information to estimate $S_{I}$ in this subject. All in all, it is difficult to tell whether the $\mathrm{S}_{\mathrm{I}}$ of subject 3 is really higher than that of subject 2. The examination of the $S_{1}^{\mathrm{D}}$ results obtained in the same subjects offers a completely different scenario. In fact, the marginal posteriors of $S_{1}^{D}$ (third column of Fig. 1), are well concentrated around their means in both subjects. The $S_{1}^{\mathrm{D}}$ point estimates are $1.17(1.1-1.23)$ and $0.06(0.028-0.1)$, respectively, and this suggests that insulin is in all likelihood more effective to enhance glucose disappearance in subject 2 than in subject 3 . To understand why the point estimate of $S_{1}^{\mathrm{D}}$ is much
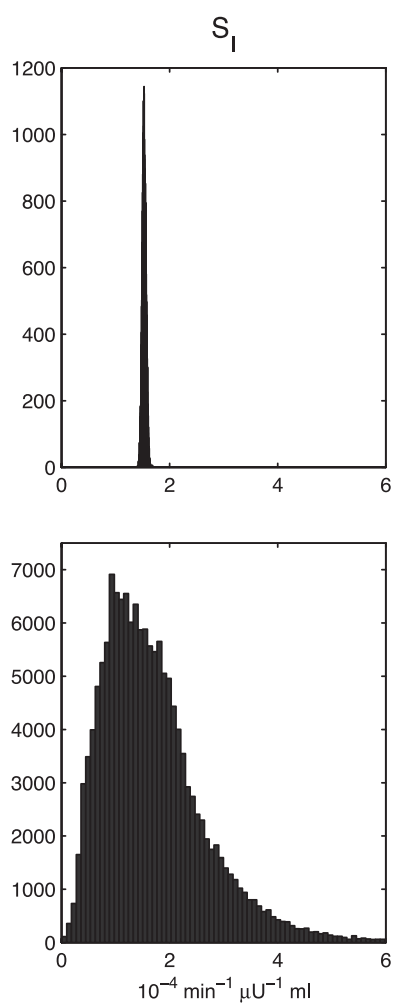
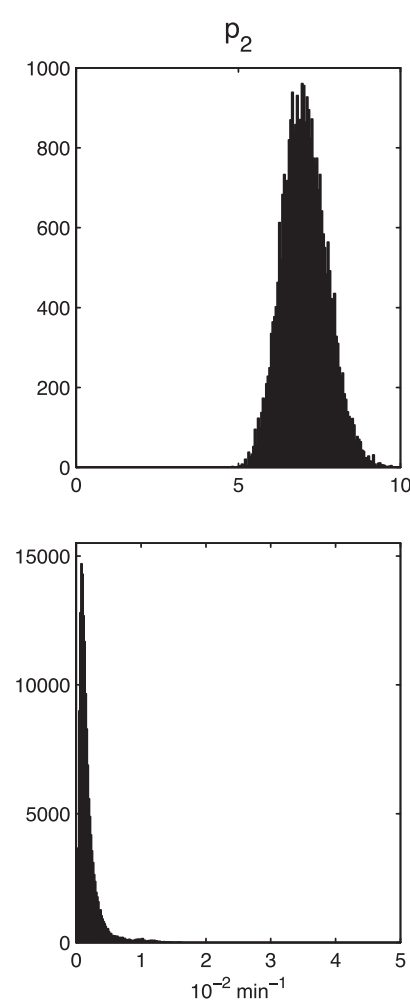
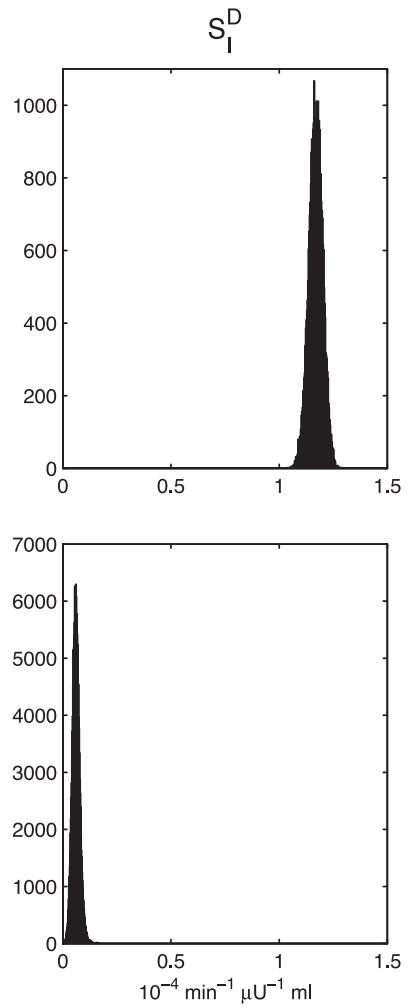

Fig. 1. Intravenous glucose tolerance test (IVGTT): diabetic subjects 2 (top) and 3 (bottom). Marginal posterior [obtained in sampled form by Markov Chain Monte Carlo $(\mathrm{MCMC})]$ of the index of insulin sensitivity $\left(\mathrm{S}_{\mathrm{I}} ;\right.$ left), parameter $p_{2}$ (middle), and new dynamic insulin sensitivity index $\left(\mathrm{S}_{\mathrm{I}}^{\mathrm{D}}\right.$; right). 
Fig. 2. IVGTT: Bayes $S_{I}$ and $S_{I}^{D}$ in diabetic subjects. Point estimates are shown with the $95 \%$ confidence intervals (horizontal bars).

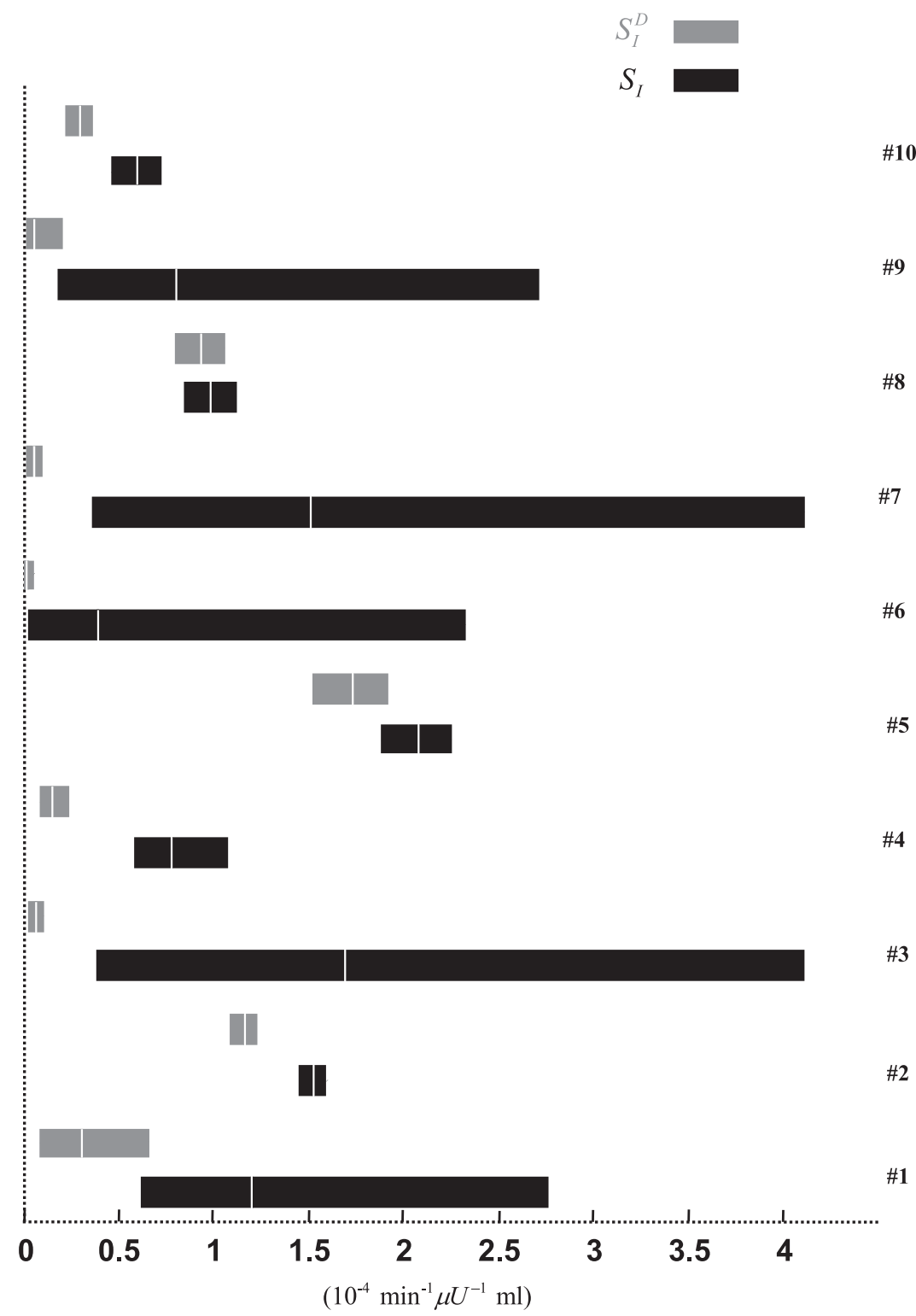

\#9

\#8

$\# 7$

\#6

lower in subject 3 than in subject 2, one must consider that $S_{1}^{\mathrm{D}}$ embodies the information brought about by both $\mathrm{S}_{\mathrm{I}}$ and $p_{2}$ (the latter parameter is reported in the second column of Fig. 1). It can be seen that in subject 3 the $S_{I} 95 \%$ confidence interval includes many $S_{I}$ values that are coupled with very low values of $p_{2}$. This means that in this subject $\mathrm{S}_{\mathrm{I}}$ is frequently associated with very low values of the efficiency $\eta$, which translates into a low point estimate of $S_{1}^{\mathrm{D}}$.

The usefulness of the new index is also apparent when the whole group of diabetic subjects is considered. The results reported in Fig. 2 displaying the $S_{I}$ and $S^{D}$ estimates together with their 95\% confidence intervals indicate that $S_{1}^{D}$ never exhibited numerical identifiability problems. In contrast, the $S_{I}$ results found in subjects $1,3,6,7$, and 9 are rather difficult to interpret.

IVGTT in diabetic subjects: Bayes vs. Fisher estimation of $S_{I}^{D}$. In Ref. 19, the Bayes approach was shown to be much more robust than the Fisher one in estimating $\mathrm{S}_{\mathrm{I}}$ and $p_{2}$ in NGT subjects. Bayes superiority over Fisher can be even more appreciated in the present study because diabetic patients exhibit low values of $S_{I}$ that are often coupled with low values of $p_{2}$. In this case, the profile of insulin action becomes so low and slow so as to degrade the precision of the MM parameter estimates. In fact, ML often yields an estimate of $S_{I}$ that is affected by a large uncertainty and cannot be discriminated from zero (this is known in the literature as the " $\mathrm{S}_{\mathrm{I}}=0$ " problem; Refs. 1, 18, 19). As a consequence, the histogram describing the $S_{I}$ frequency distribution is bimodal showing an artifactual peak at $S_{I}=0$ and another peak at a positive $S_{I}$ value, which leads to interpretative difficulties. Another problem is also that $S_{I}$ estimates can turn out to be unrealistically high. Bayes estimation, implemented by using MCMC, is more robust than Fisher estimation since it provides a nonzero minimum variance estimate of $S_{I}$ that hinges on the entire marginal posterior of $\mathrm{S}_{\mathrm{I}}$. In addition, rigorous confidence intervals for $S_{\mathrm{I}}$ can be calculated. The question arises as to whether the same results also hold for $S_{1}^{\mathrm{D}}$. Since $S_{1}^{\mathrm{D}}$ depends on both $p_{2}$ and $\mathrm{S}_{\mathrm{I}}$ (see $E q .5$ ), one would not be surprised to find 

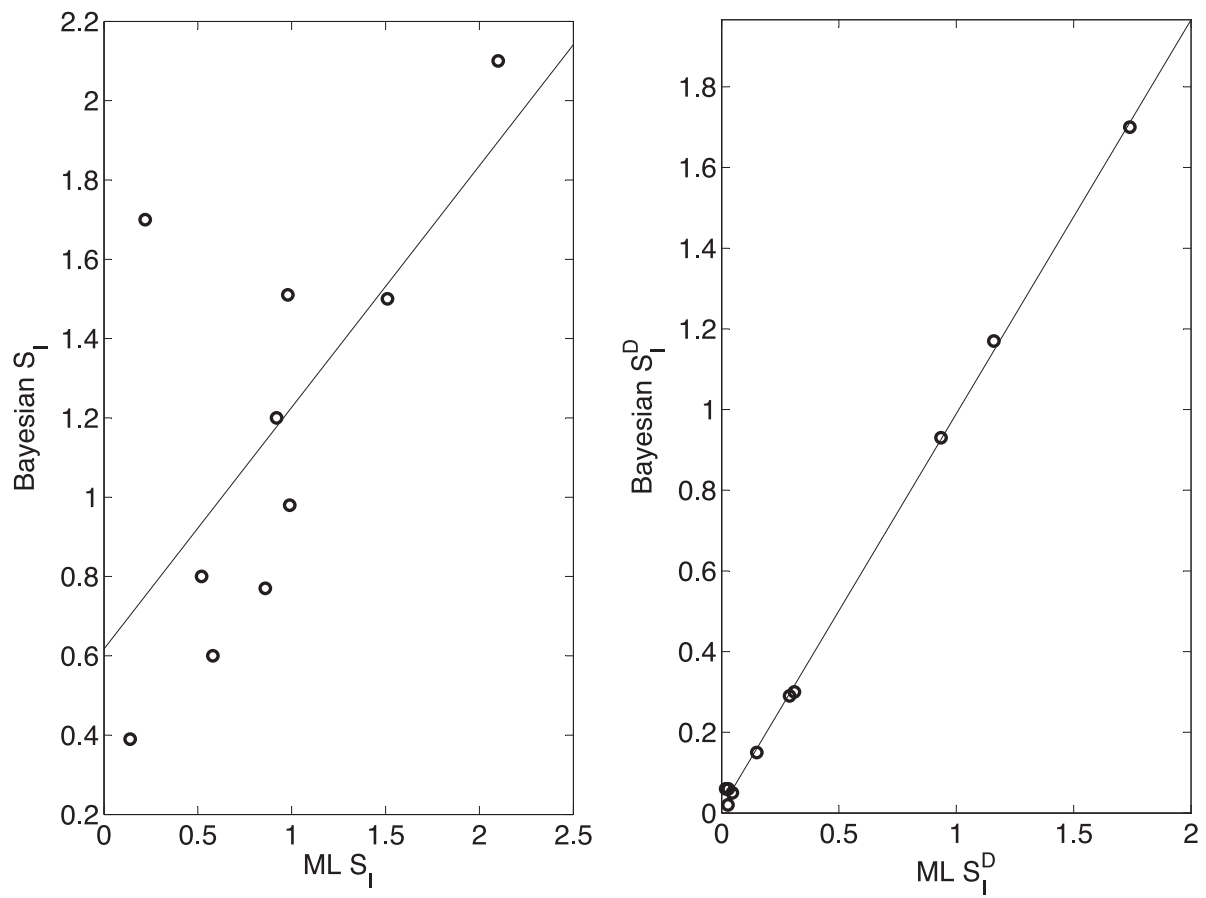

Fig. 3. IVGTT. Left: maximum likelihood vs. Bayesian $\mathrm{S}_{\mathrm{I}}$ estimates in diabetic subjects. Right: ML vs. Bayesian $S_{1}^{D}$ in diabetic subjects.

out that also the Fisherian point estimate of $\mathrm{S}_{\mathrm{I}}^{\mathrm{D}}$ is plagued by the same problem described above for $S_{I}$. Fortunately, however, the Fisherian estimate of $S_{I}^{D}$ was often very close to the one obtained with the Bayesian approach. This can be appreciated by looking at Fig. 3 where the Bayesian and Fisherian estimates of $\mathrm{S}_{\mathrm{I}}$ a $\mathrm{S}_{\mathrm{I}}^{\mathrm{D}}$ are reported. As said, the correlation coefficient between ML and Bayes estimates of $S_{I}$ and of $\mathrm{S}_{\mathrm{I}}^{\mathrm{D}}$ was 0.67 and 0.99 , respectively. This favorable outcome for $\mathrm{S}_{\mathrm{I}}^{\mathrm{D}}$ is related with the functional relationships among $\mathrm{S}_{\mathrm{I}}, p_{2}$, IAIR, and $S_{I}^{D}$. While it is difficult to estimate precisely $S_{I}$ and $p_{2}$, due to the presence of numerical nonidentifiability regions that make many different combinations of $\mathrm{S}_{\mathrm{I}}$ and $p_{2}$ capable of generating virtually the same IAIR on a finite time interval, IAIR itself and its integral (and thus $\mathrm{S}_{\mathrm{I}}^{\mathrm{D}}$ ) can be precisely determined. Thus, although Bayes is more robust in estimating $\mathrm{S}_{\mathrm{I}}$ and $p_{2}$ separately, Bayes and Fisher behave similarly as regards $S_{I}^{D}$.
In summary, the Fisher estimator often provides $S_{I}^{D}$ estimates comparable to the Bayesian ones with a reduced computational effort (few seconds in place of minutes, since Markov chain generation and Monte Carlo integration are more demanding than mode-finding algorithms). This advantage of $\mathrm{S}_{\mathrm{I}}^{\mathrm{D}}$ over $\mathrm{S}_{\mathrm{I}}$ may be particularly relevant in large-scale studies in which many model identifications must be carried out.

IVGTT and MGTT in diabetic subjects: sensitivity of $S_{I}$ and $S_{I}^{D}$ to prior. To assess the sensitivity of the results to the prior information on $\mathrm{S}_{\mathrm{I}}$ used in the MM Bayesian identification from IVGTT in diabetics, the estimation process was repeated using larger thresholds ( 3 and 4 in place of 2). As already found in Ref. $19, \mathrm{~S}_{\mathrm{I}}$ and $p_{2}$ were particularly sensitive to prior information. In particular, the prior information exerted a profound influence on the tails of the $\mathrm{S}_{\mathrm{I}}$ and $p_{2}$ marginal posteriors. Quite to the contrary, we found (results not shown) that the new index $S^{D}$ is scarcely sensitive to the prior information. MM

Table 3. MGTT in diabetic subjects: $S_{I}, S_{I}^{D}$, and $p_{2}$ estimates obtained using prior information on GEZI

\begin{tabular}{|c|c|c|c|c|c|c|}
\hline Subject Nos. & $\mathrm{S}_{\mathrm{I}}(1)$ & $\mathrm{S}_{\mathrm{I}}(2)$ & $\mathrm{S}_{\mathrm{I}}^{\mathrm{D}}(1)$ & $\mathrm{S}_{\mathrm{I}}^{\mathrm{D}}(2)$ & $p_{2}(1)$ & $p_{2}(2)$ \\
\hline 2 & 2.0 & 2.0 & 0.41 & 0.39 & 0.78 & 0.758 \\
\hline 3 & 6.8 & 6.6 & 0.73 & 0.74 & 0.38 & 0.404 \\
\hline 5 & 2.1 & 4.3 & 0.16 & 0.14 & 0.258 & 0.116 \\
\hline 6 & 4.4 & 6.5 & 0.349 & 0.33 & 0.28 & 0.177 \\
\hline 7 & 1.5 & 1.38 & 0.55 & 0.23 & 1.7 & 0.63 \\
\hline 11 & 4.6 & 0.8 & 0.17 & 0.17 & 0.13 & 0.053 \\
\hline 12 & 5.8 & 2.5 & 0.42 & 0.39 & 0.26 & 0.107 \\
\hline 13 & 9.9 & 20 & 0.31 & 0.3 & 0.107 & 0.05 \\
\hline 14 & 24.1 & 24.3 & 0.0064 & 0.0057 & 0.00088 & 0.00078 \\
\hline
\end{tabular}

Estimates are denoted by $\mathrm{S}_{\mathrm{I}}(1), \mathrm{S}_{\mathrm{I}}^{\mathrm{D}}(1)$, and $p_{2}(1)$ when using the full prior and by $\mathrm{S}_{\mathrm{I}}(2), \mathrm{S}_{\mathrm{I}}^{\mathrm{D}}(2)$, and $p_{2}(2)$ when using the less informative prior; GEZI, glucose effectiveness at zero insulin; MGTT, meal glucose tolerance test; measurement units of MM purameters are the same as in Tables 1 and 2. 
Fig. 4. Meal glucose tolerance test (MGTT). Left: insulin sensitivity using full prior information on glucose effectiveness at zero insulin [GEZI; $\left.\mathrm{S}_{\mathrm{I}}(1)\right]$ vs. less informative $\left[\mathrm{S}_{\mathrm{I}}(2)\right]$. Right: dynamic insulin sensitivity using full prior information on GEZI $\left[\mathrm{S}_{\mathrm{I}}^{\mathrm{D}}(1)\right]$ vs. less informative $\left[\mathrm{S}_{\mathrm{I}}^{\mathrm{D}}(2)\right]$.
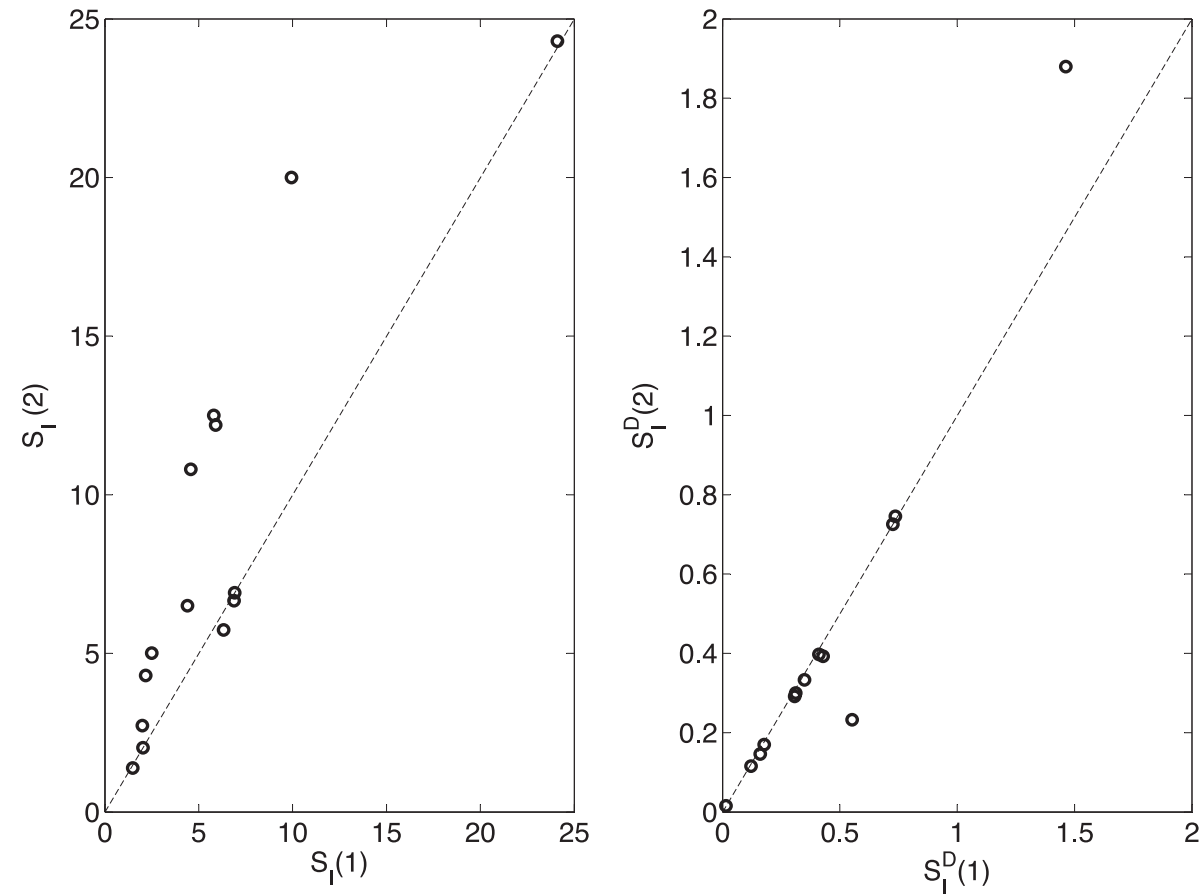

identification from MGTT data in the 14 diabetic patients provides further support to these findings. In fact, inspection of Table 3 and Fig. 4 indicate that, at variance with $S_{I}$ and $p_{2}$, the estimates of $S_{I}^{D}$ are virtually insensitive to the prior information on GEZI.

The reasons for the higher robustness of $S_{1}^{\mathrm{D}}$ with respect to $\mathrm{S}_{\mathrm{I}}$ and $p_{2}$ are the same already put forth to discuss the Bayesian vs. Fisherian identification results. The parameters $\mathrm{S}_{\mathrm{I}}$ and $p_{2}$ are more sensitive to the prior information because of the existence of numerical nonidentifiability regions in the param- eter space where changes in these two parameters only produce negligible changes in the likelihood (18). Within such numerical nonidentifiability regions, many different combinations of $\mathrm{S}_{\mathrm{I}}$ and $p_{2}$ may lead to similar IAIR. Since $\mathrm{S}_{1}^{\mathrm{D}}$ is the mean of IAIR in the interval $(0, T)$ with $T=60 \mathrm{~min}$, many different combinations of $S_{I}$ and $p_{2}$ are mapped into the same $S_{1}^{D}$. Therefore, whereas $\mathrm{S}_{\mathrm{I}}$ and $p_{2}$ may be very uncertain, $\mathrm{S}_{1}^{\mathrm{D}}$ is not. This peculiarity of $S_{I}^{\mathrm{D}}$ can be easily grasped by resorting to a clamp thought experiment, where $\mathrm{S}_{\mathrm{I}}$ depends on the asymptotic value of the exogenous glucose infusion. Large and unrealistic
Fig. 5. Left: true $S_{I}$ vs. estimated $S_{I}$ in diabetic (top) and NGT subjects (bottom). Right: true $\mathrm{S}_{\mathrm{I}}^{\mathrm{D}}$ vs. estimated $\mathrm{S}_{\mathrm{I}}^{\mathrm{D}}$ in diabetic (top) and NGT subjects (bottom).
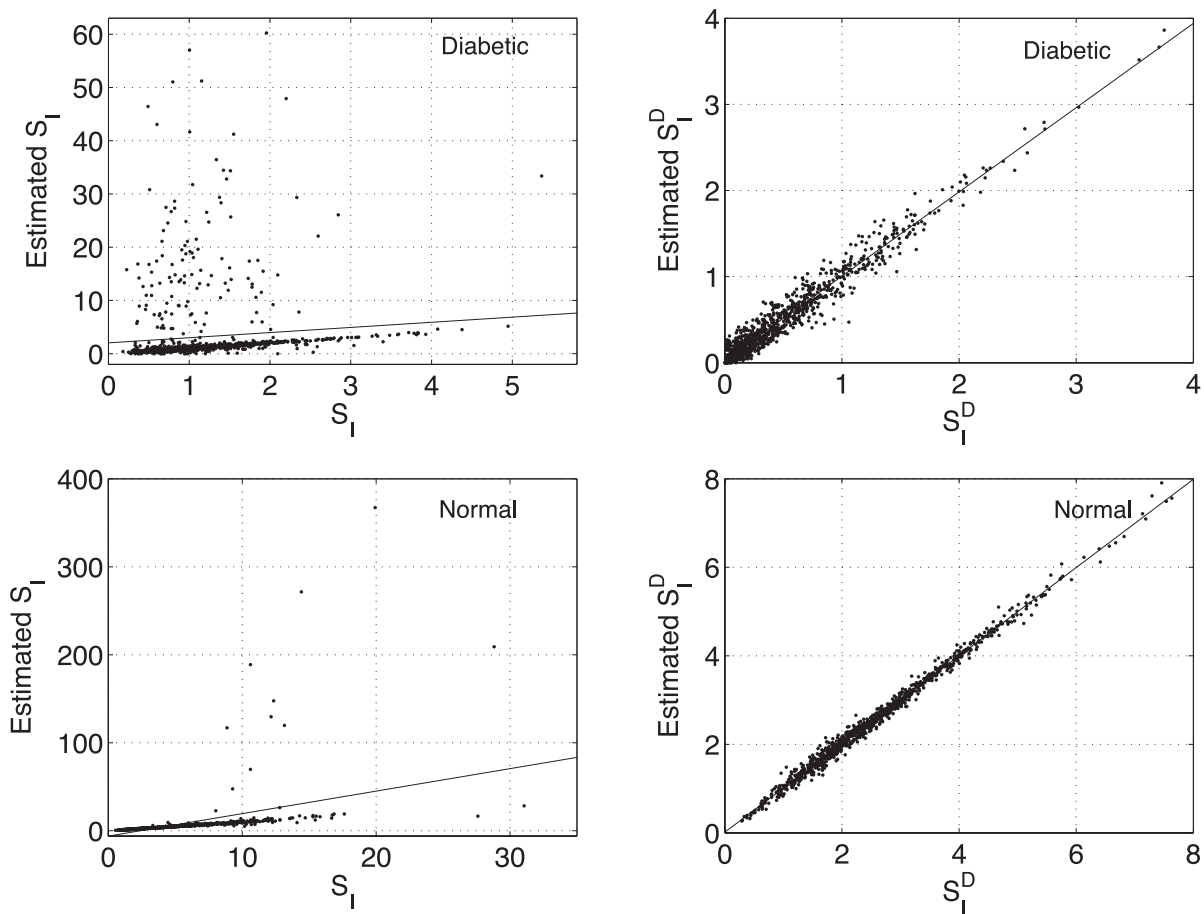


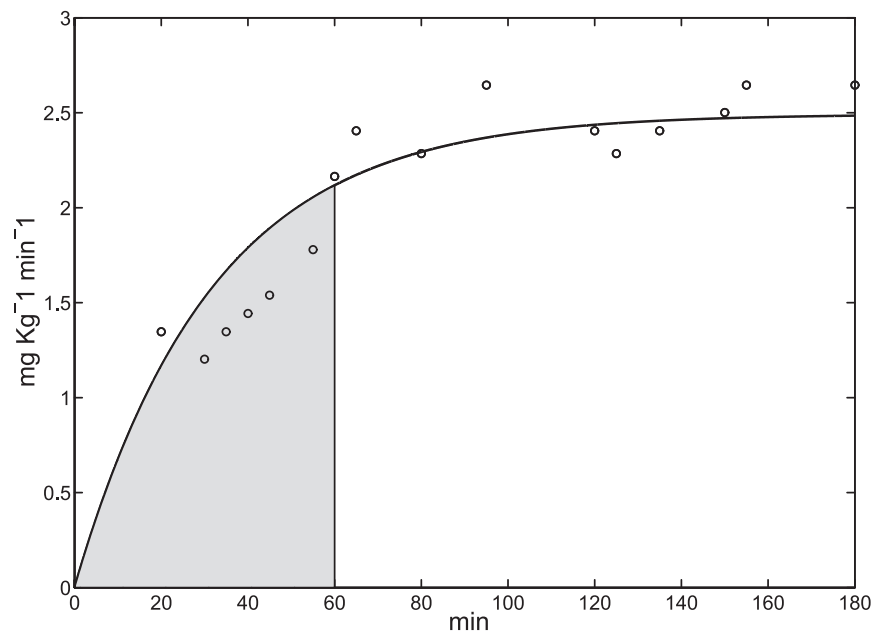

Fig. 6. Clamp model. Samples of glucose infusion measured in the first IGT subject (circles) and curve (proportional to IAIR) obtained after fitting the samples using Eq. 8 (solid line). Area under the curve in the first $60 \mathrm{~min}$ is proportional to $\mathrm{S}_{\mathrm{I}}^{\mathrm{D}}$ (clamp).

$\mathrm{S}_{\mathrm{I}}$ values may be obtained in diabetic subjects since they are associated with low $p_{2}$ values that define virtual glucose infusions similar to straight lines, which "almost diverge" at infinity. On the other hand, by definition, $S_{I}^{D}$ is proportional to the area of glucose infusion in the first $60 \mathrm{~min}$ and it is thus much less sensitive to poor numerical identifiability of $p_{2}$. As a practical consequence, $S_{I}^{D}$ can be reliably estimated from IVGTT/MGTT experiments also under conditions where $\mathrm{S}_{\text {I }}$ and $p_{2}$ suffer from poor numerical identifiability. The small sensitivity of $S^{D}$ to the prior information contributes to make $\mathrm{S}_{\mathrm{I}}^{\mathrm{D}}$ a much more robust and objective measure of insulin ability to control glucose.

IVGTT: $S_{I}$ vs. $S_{I}^{D}$ ability in discriminating NGT and diabetic subjects. The results reported in Fig. 5 provide further support to the notion that $S_{I}^{D}$ can be estimated with much more precision than $S_{\text {I }}$ during an IVGTT experiment, especially when diabetic subjects are under study. As already said, the correlation coefficients between true and estimated $S_{\text {I }}$ are $\sim 0.1$ and 0.4 in diabetic and NGT subjects, respectively, while those concerning $\mathrm{S}_{\mathrm{I}}^{\mathrm{D}}$ are 0.98 and 0.99 , respectively. It is of interest that in Fig. 5, top left, almost $10 \%$ of estimated $\mathrm{S}_{\mathrm{I}}$ values turn out to be 10 times larger than the true ones. This means that, in a nonnegligible number of cases, a diabetic patient is erroneously classified as a subject with a normal insulin sensitivity. In contrast, the discriminatory power of $\mathrm{S}^{\mathrm{D}}$ is always excellent. This new evidence of the superior performance of $S_{I}^{D}$ against $S_{I}$ adds to the one previously reported in Refs. 17 and 20, where we showed that the new dynamic index is better correlated than $\mathrm{S}_{\text {I }}$ with the time by which glucose concentration reattains its basal level after a glucose perturbation.

Validation of $S_{I}^{D}$ against the euglycemic hyperinsulinemic clamp. Figure 6 shows the area under the curve in the first 60 min of the clamp experiment, proportional to $S_{I}^{D}$ (clamp). This clearly shows how the new index measures the ability of insulin to control glucose by accounting not only for the final value achieved by the glucose infusion but also for the speed of rise of the glucose infusion.

The results displayed in Fig. 7 show that the OGTT-based estimates of $\mathrm{S}_{\mathrm{I}}^{\mathrm{D}}$ and $\mathrm{S}_{\mathrm{I}}^{\mathrm{D}}($ clamp) are well correlated $(r=0.82)$.
Such degree of correlation is almost identical to the one between $\mathrm{S}_{\mathrm{I}}$ and $\mathrm{S}_{\mathrm{I}}$ (clamp) $(r=0.81)$ obtained from the analysis of the same experimental data in a previous report (9). It is worth noticing that if we focus our attention on the results obtained in the 11 IGT subjects, the correlation index between $\mathrm{S}_{\mathrm{I}}^{\mathrm{D}}$ and $\mathrm{S}_{\mathrm{I}}^{\mathrm{D}}$ (clamp) is 0.91 , whereas that between $\mathrm{S}_{\mathrm{I}}$ and $\mathrm{S}_{\mathrm{I}^{-}}$ (clamp) is only 0.7 . Instead, in the $10 \mathrm{NGT}$ subjects, the two correlation indexes are similar and $\sim 0.6$. This dissimilarity also points in the direction that the benefits of $\mathrm{S}_{\mathrm{I}}^{\mathrm{D}}$ become more and more relevant as the degree of glucose tolerance decreases.

\section{Conclusions}

The present study is a follow up of previous paper in NGT subjects (17) where we pointed out the limitations of the classical MM index of insulin sensitivity, $\mathrm{S}_{\mathrm{I}}$, and proposed a novel index, $\mathrm{S}_{\mathrm{I}}^{\mathrm{D}}$. The new index yields a more comprehensive picture of insulin's ability to control glucose metabolism because it hinges upon a theoretically based combination of $p_{2}$ and $p_{3}$ that better accounts for the dynamic properties of insulin action. For instance, $\mathrm{S}_{\mathrm{I}}^{\mathrm{D}}$ has been shown to be better correlated than $S_{I}$ with the time by which glucose concentration reattains its basal level after a glucose bolus (17). Further, by incorporating the concept of efficiency, the new index provides an elegant answer to those perplexing situations in which, despite an elevated value of $\mathrm{S}_{\mathrm{I}}$, the rate of glucose disappearance seems to be scarcely affected by insulin. This occurs when the elevated $S_{I}$ cannot be promptly translated into effective insulin action due to a low $p_{2}$ value. This situation is exemplified by the results of patient 14 in Table 3 . In fact, in this subject, the large $S_{I}$ value would suggest that insulin control is in the normal range, whereas the $S_{I}^{D}$ value unmasks a low ability to restore glucose homeostasis. The simulation study results reported in Fig. 5 further corroborate the notion that $\mathrm{S}^{\mathrm{D}}$ can considerably improve the reliability of the assessment of insulin sensitivity in diabetic patients. Furthermore, the issue of the validity of $\mathrm{S}_{\mathrm{I}}^{\mathrm{D}}$ against a reference measure has been tackled in the present study by comparing the estimates of $S^{D}$ obtained from an OGTT with those obtained in the same subjects from a euglycemic hyperinsulinemic clamp, i.e., the gold standard technique for the assessment of insulin sensitivity.

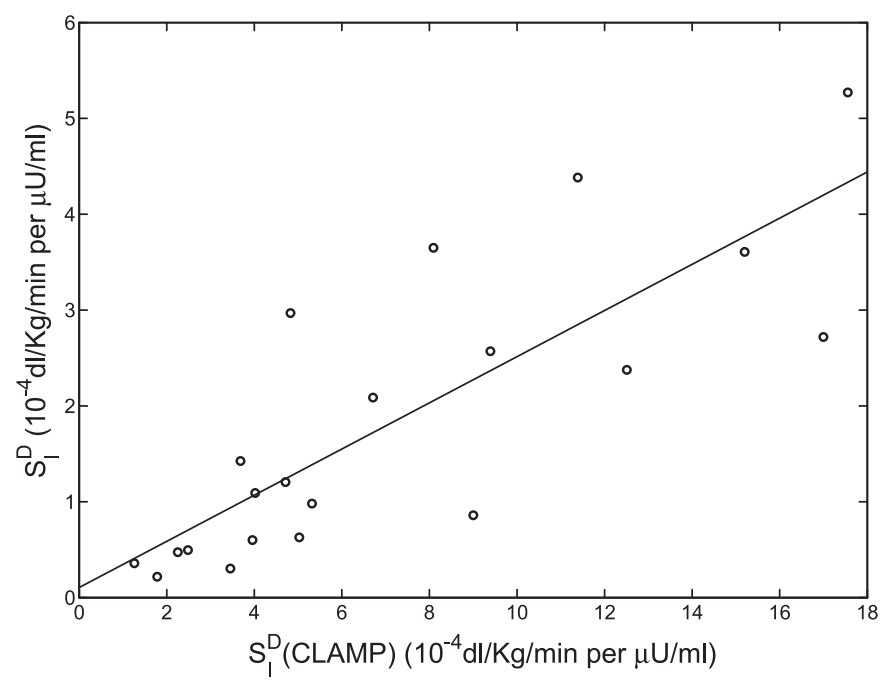

Fig. 7. Clamp and oral minimal model. $S_{\mathrm{I}}^{\mathrm{D}}$ vs. $\mathrm{S}_{\mathrm{I}}^{\mathrm{D}}$ (clamp) estimates in IGT and NGT subjects. 
The results of the present study indicate that the new dynamic index is especially useful in those diabetic subjects having a slow timing of insulin action. It is well known that, when analyzing IVGTT data, the estimation of $p_{2}$ in diabetic subjects is often problematic. One of the most relevant findings of the present study is that $S^{D}$ is much less affected than $S_{I}$ by such a drawback. Thus $S_{I}^{D}$ has the additional advantage over $S_{I}$ in that its estimation is much more robust and can be accomplished by resorting to the Fisher approach. In fact, at variance with what happens with $\mathrm{S}_{\mathrm{I}}$, the Fisherian and Bayesian point estimates of $S^{D}$ are similar, with the latter being virtually insensitive to the prior information incorporated in the estimator. Since ML is much less computationally demanding than MCMC, this makes $S_{I}^{D}$ more appealing than $S_{I}$ in studies where many subjects have to be analyzed. $S^{D}$ thus appears particularly suitable to be employed in population studies where glucose intolerant subjects are likely to be present, as well as in studies seeking for diabetes-relevant genes in the human genome $(12,14,21,22)$.

\section{GRANTS}

This work was in part supported by National Institute of Biomedical Imaging and Bioengineering EB-001975 and by the PRIN Project "New Methods and Algorithms for Identification and Adaptive Control of Technological Systems."

\section{DISCLOSURES}

No conflicts of interest are declared by the author(s).

\section{REFERENCES}

1. Avogaro A, Vicini P, Valerio A, Caumo A, Cobelli C. The hot but not the cold MM allows precise assessment of insulin sensitivity in NIDDM subjects. Am J Physiol Endocrinol Metab 270: E532-E540, 1996.

2. Barrett PHR, Bell BM, Cobelli C, Golde H, Schumitzky A, Vicini P, Foster D. SAAMII: simulation, analysis and modeling software for tracer and pharmacokinetic studies. Metabolism 47:484-492, 1998.

3. Basu A, Dalla Man C, Basu R, Toffolo G, Cobelli C, Rizza RA. Effects of type 2 diabetes on insulin secretion, insulin action, glucose effectiveness, and postprandial glucose metabolism. Diabetes Care 32: 866-872, 2009.

4. Bergman RN, Ider YZ, Bowden CR, Cobelli C. Quantitative estimation of insulin sensitivity. Am J Physiol Endocrinol Metab 236: E667-E677, 1979.

5. Bergman RN, Bowden CR, Cobelli C. The MM approach to quantification of factors controlling glucose disposal in man. In: Carbohydrate Metabolism, edited by Cobelli C and Bergman RN. New York: Wiley, 1981.
6. Braun M. Differential Equations and Their Applications. New York: Springer-Verlag, 1993.

7. Carson ER, Cobelli C, Finkenstein L. The Mathematical Modeling of Metabolic and Endocrine Systems. New York: Wiley, 1983.

8. Dalla Man C, Caumo A, Cobelli C. The oral glucose MM: estimation of insulin sensitivity from a meal test. IEEE Trans Biomed Eng 49: 419-429, 2002.

9. Dalla Man C, Yarasheski KE, Caumo A, Robertson H, Toffolo G, Polonsky KS, Cobelli C. Insulin sensitivity by oral glucose MMs: validation against clamp. Am J Physiol Endocrinol Metab 289: E954E958, 2005.

10. Gelman A, Carlin JB, Stern HS, Rubin DB. Bayesian Data Analysis. London: Chapman \& Hall, 1995.

11. Gilks WR, Richardson S, Spiegelhalter DJ. Markov Chain Monte Carlo in Practice. London: Chapman \& Hall, 1996.

12. Haffner SM, D'Agostino R Jr, Mykkanen L, Tracy R, Howard B, Rewers M, Selby J, Savage PJ, Saad MF. Insulin sensitivity in subjects with type 2 diabetes. Relationship to cardiovascular risk factors: the Insulin Resistance Atherosclerosis Study. Diabetes Care 22: 562-568, 1999.

13. Hastings WK. Monte Carlo sampling methods using Markov chain and their applications. Biometrika 57: 97-109, 1970.

14. Mykkanen L, Zaccaro DJ, Hales CN, Festa A, Haffner SM. The relation of proinsulin and insulin to insulin sensitivity and acute insulin response in subjects with newly diagnosed type II diabetes: the Insulin Resistance Atherosclerosis Study. Diabetologia 42: 1060-1066, 1999.

15. Nolan JJ, Ludvik B, Baloga J, Reichart D, Olefsky JM. Mechanisms of the kinetic defect in insulin action in obesity and NIDDM. Diabetes 46: 994-1000, 1997.

16. Owens DR, Luzio SD, Coates PA. Insulin secretion and sensitivity in newly diagnosed NIDDM caucasians in the UK. Diabet Med 13: S19S24, 1996.

17. Pillonetto G, Caumo A, Sparacino G, Cobelli C. A new dynamic index of insulin sensitivity. IEEE Trans Biomed Eng 53: 369-379, 2006.

18. Pillonetto G, Sparacino G, Cobelli C. Numerical non-identifiability regions of the MM of glucose kinetics: superiority of Bayesian estimation. Math Biosci 184: 53-67, 2003

19. Pillonetto G, Sparacino G, Magni P, Bellazzi R, Cobelli C. Minimal model $S_{I}=0$ problem in NIDDM subjects: nonzero Bayesian estimates with credible confidence intervals. Am J Physiol Endocrinol Metab 282: E564-E573, 2002.

20. Pillonetto G, Cobelli C. Predictive power of indices derived from models of biological dynamic systems. Proceed Am Contr Conf, 2008, p. 21242129, 2008.

21. Raffael JR, Robbins DC, Norris JM, Boerwinkle E, Defronzo RA, Elbein SC, Fujimoto W, Hanis CL, Kahn SE, Permutt MA, Chiu KC, Cruz J, Ehrmann DA, Robertson RP, Rotter JI, Buse J. The GENNID study: a resource for mapping the genes that cause NIDDM. Diabetes Care 19: 864-872, 1996.

22. Saad MF, Anderson RL, Laws A, Watanabe RM, Kades WW, Chen YDI, Sands RE, Pei D, Savage PJ, Bergman RN. A comparison between the MM and the glucose clamp in the assessment of insulin sensitivity across the spectrum of glucose tolerance. Diabetes 43: 1114-1121, 1994. 